Published in final edited form as:

Curr Opin Chem Eng. 2014 February ; 3: 112-117. doi:10.1016/j.coche.2013.12.008.

\title{
TISSUE ENGINEERING PERFUSABLE CANCER MODELS
}

\author{
E.L. Fong ${ }^{\mathrm{a}, \S}$, M. Santoro ${ }^{\mathrm{b}} \S$, M.C. Farach-Carson ${ }^{\mathrm{a}, \mathrm{c}}$, F.K. Kasper $^{\mathrm{a}}$, and A.G. Mikos ${ }^{\mathrm{a}, \mathrm{b},{ }^{\star}}$ \\ aDepartment of Bioengineering, Rice University, Houston, TX 77030 \\ ${ }^{b}$ Department of Chemical and Biomolecular Engineering, Rice University, Houston, TX 77005 \\ 'Department of Biochemistry and Cell Biology, Rice University, Houston, TX 77251
}

\begin{abstract}
The effect of fluid flow on cancer progression is currently not well understood, highlighting the need for perfused tumor models to close this gap in knowledge. Enabling biological processes at the cellular level to be modeled with high spatiotemporal control, microfluidic tumor models have demonstrated applicability as platforms to study cell-cell interactions, effect of interstitial flow on tumor migration and the role of vascular barrier function. To account for the multi-scale nature of cancer growth and invasion, macroscale models are also necessary. The consideration of fluid dynamics within tumor models at both the micro- and macroscopic levels may greatly improve our ability to more fully mimic the tumor microenvironment.
\end{abstract}

\section{Keywords}

Tissue engineering; Cancer; Perfusion; Tumor models; Fluid flow

\section{Introduction}

In light of the inherent limitations of traditional two-dimensional (2D) tissue culture systems and animal models for preclinical drug testing and mechanistic studies, considerable progress has been made in the development of ex vivo three-dimensional (3D) tumor models that more fully recapitulate aspects of the in vivo tumor niche.[1,2] While the human tumor spheroid is currently the most commonly used 3D model, increasingly, enabling technologies developed in the tissue-engineering field are being leveraged to construct models that mimic the tumor microenvironment with greater fidelity. While these 3D models have appreciably advanced our understanding of the effects that the tissue architecture, extracellular matrix (ECM), and tumor-stroma interaction have on tumorigenesis, the impact of a generally neglected but significant component of the tumor microenvironment - fluid flow - remains largely undocumented.[3] Due to rapid and abnormal tumor-associated angiogenesis and the formation of a leaky and aberrant vasculature [4], the permeability of water and solutes within a tumor increases, resulting in a rise in interstitial fluid pressure and corresponding net convective flow out from the tumor mass into the surrounding tissue. [5,6] This interstitial fluid flow not only results in shear

(C) 2014 Elsevier Ltd. All rights reserved.

*To whom correspondence may be addressed: A.G. Mikos, PhD, Department of Bioengineering, MS-142, BioScience Research Collaborative, Rice University, 6500 Main Street, Houston, TX 77030, mikos@ rice.edu; Tel: (713) 348-5355, Fax: (713) 348-4244.

$\S$ These authors contributed equally to this work

Publisher's Disclaimer: This is a PDF file of an unedited manuscript that has been accepted for publication. As a service to our customers we are providing this early version of the manuscript. The manuscript will undergo copyediting, typesetting, and review of the resulting proof before it is published in its final citable form. Please note that during the production process errors may be discovered which could affect the content, and all legal disclaimers that apply to the journal pertain. 
forces which may influence tumor cell proliferation [7], but also creates extracellular gradients of proteases and cytokines that may promote cancer invasion. [5,8-10]

Despite the increasing realization that fluid flow is an integral part of the tumor microenvironment, the contribution of this component to cancer progression remains to be fully elucidated. Notably, besides the need to better understand the repercussions of fluid flow in the tumor microenvironment, the inclusion of perfusion is also a potential strategy to overcome the diffusional limitations associated with most 3D tumor models.[1] To achieve this goal of recapitulating tumor-associated fluid dynamics, tight integration of the two most relevant engineering disciplines, microfluidics and tissue engineering, is necessary. This review will cover the current status in the development of microfluidic as well as macroscale tissue-engineered tumor models, and discuss the challenges involved in taking an interdisciplinary approach towards the development of perfused tissue-engineered tumor models.

\section{Microfluidic Tumor Models}

Since their advent, microfluidic systems have garnered the attention of the oncological community due to the possibility of modeling varying aspects of tumorigenesis characterized by a highly controlled spatiotemporal development, such as intercellular communication, metastasis and drug resistance. The laminar flow condition arising at the micro-scale presents the opportunity to mimic concentration gradients typical of the tumor niche [11,12], with better recapitulation of drug transport and transient biological processes occurring down to the single cell level.[13] This unique property, together with the potential for high-throughput and automation, has been leveraged in the last decade mainly for proteomic analyses [14], cell sorting [15], and pharmacodynamics studies [16], ultimately projecting microfluidic systems as highly advanced analytical tools for tumor biology research. One of the most prominent manifestations of this research direction is represented by the work of Jang et al., who developed a platform able to evaluate up to 100 different drug combinations, in line with the emerging importance of combinatorial drug therapy for chemoresistant patients.[17] The development of microfabrication techniques such as microcontact printing and soft lithography further enhanced microfluidic capabilities, that is, better control over topology and surface chemistry,[18] hence paving the way for more sophisticated mechanistic investigations of tumor biology.[13,19,20] Several groups have leveraged the preferential adhesion of endothelial cells and tumor cells on fibronectin and hyaluronic acid, respectively, to investigate tumor-vasculature interactions on microcontactprinted scaffolds with a particular focus on cancer cell motility associated with extravasation and metastasis.[21,22]

The ultimate advancement in the applicability of microfluidics to oncology stems from the use of 3D scaffolds to more fully recapitulate the native tumor niche and incorporate the effects of matrix composition, structure and mechanical properties on tumor progression. $[19,23,24]$ Mirroring the current trend in macroscale tumor models, biocompatible hydrogels such as collagen [25], alginate [24], and Matrigel ${ }^{\mathrm{TM}}$ [26], are popular scaffolds used to investigate the role of cell-cell, cell-ECM and hydrodynamic cues on tumor progression and drug efficacy within a microfluidic context. In one example illustrating the precise spatial control that can be achieved with microfluidic systems, Huang et al. demonstrated the ability to pattern separate, adjacent channels with distinct hydrogel types.[27] With this microfluidic configuration, tumor-derived macrophage cells encapsulated within Matrigel ${ }^{\mathrm{TM}}$ were observed to invade into contiguous metastatic breast cancer cell - laden collagen hydrogels and not blank gels, highlighting the potential utility of this system for systematic studies of cell-cell and cell-ECM interactions. In a similar compartmentalized platform, Sung et al. demonstrated the distance-dependent effects of breast cancer progression towards 
the invasive phenotype using a ' $Y$ '-shaped channel in a co-culture model of mammary epithelial cells and fibroblasts.[26]

Beyond being able to mimic the multicellular cross-talk within the tumor microenvironment, microfluidic culture systems have recently been shown to have utility in investigating the effect of interstitial flow on tumor cell migration. Using a microfluidic device consisting of two channels separated by a 3D collagen I matrix seeded with breast carcinoma cells, Polacheck et al. demonstrated the feasibility of generating tumor-relevant interstitial flow via the application of a hydrostatic pressure gradient across the construct.[28] This study demonstrated how the directional bias of migration along the streamline is determined not only by cell density, but also the interstitial flow rate. Interestingly, as the tendency for upstream migration of cells was found to correlate with high cell density and flow rates, the authors suggest the presence of an 'escape radius', where interstitial flow either guides tumor cells upstream to remain clustered with the tumor, or downstream towards the draining lymphatics or veins - the outcome being dependent on whether the cell is within or outside this 'escape radius'.[28] In another similar study, Haessler et al. not only demonstrated the heterogeneity of tumor cell migration in response to interstitial flow, but also the feasibility of studying the differential effects of flow on different subpopulations of cells within a heterogeneous tumor population.[29] Collectively, these mechanistic studies highlight the potential of using microfluidic culture models as tools to understand the role of interstitial flow in promoting cell migration, which may introduce novel approaches to treating metastatic disease.

A typical 'perfused' 3D cell culture system in the literature most often refers to the provision of a continuous flow of media for the supply of nutrients to and removal of wastes from the construct. However, a more precise distinction can be made to distinguish between systems where medium flows over the surface of the scaffold, as opposed to perfusion through the bulk of the scaffold.[30] Thus far, approaches to incorporate scaffold-based 3D cultures into microfluidic devices typically employ the use of hydrogels to immobilize cells for the formation of 3D structures where mass transport of solutes, primarily driven by diffusion, may not be efficient.[31] To maximize mass transfer, Toh et al. developed a microfluidic channel-based system consisting of an array of microfabricated pillars for cell immobilization and laminar flow complex coacervation reaction of polyelectrolytes for the formation of a thin layer of 3D matrix to support the cells.[32] Using carcinoma cell lines Hep G2 and MCF-7, the authors demonstrated that cells formed viable multicellular aggregates after 3 days of perfusion culture. In a similar gel-free approach to facilitate perfusion and mass transfer, the surfaces of C3A and A549 carcinoma cells were modified to contain free aldehyde groups, which reacted with an intercellular linker to form hydrazone covalent linkages between cells, facilitating cell aggregation within micropillar arrays.[33] While interesting, further investigation into the effect of fluid flow on the growth and survival of these cancer cells is warranted. Although diffusional limitations exist with the use of hydrogels as scaffolds, few in the literature have explored the possibility of using porous polymeric scaffolds to culture cancer cells within microfluidic systems. In order to evaluate the cytotoxicity of anti-cancer drugs together with liver metabolism, Ma et al. developed a micro-scale perfusion-based two-chamber system, where liver cells and glioblastoma multiforme brain cancer cells were separately cultured within two adjacent chambers housing porous poly(lactic acid) scaffolds.[34] The study demonstrated that while hepatic metabolism has a detrimental effect on the efficacy of the drug temozolomide, it is actually required for the prodrug ifosfamide to exert cytotoxicity on the cancer cells, suggesting the potential of this system for testing metabolism-dependent toxicity of anticancer drugs, an important aspect often overlooked in current in vitro systems. 
In addition to the use of microfluidic platforms to study tumor migration and drug response, another research focus has been the development of microfluidic tumor models for the study of tumor vascularization and anti-angiogenic therapy.[35] The use of endothelialized microfluidics has led to a deeper understanding of pathological vascular networks [36], elucidating the role of vascular barrier function [37], with successful description of tumor microvasculature and its effect on tumor progression.[25,38] Recent studies demonstrated the in vitro engineering of perfusable blood vessel analogues made by microfluidic channels to model and investigate tumor cell intravasation [37] and extravasation.[25] In one example, Zervantonakis et al. developed a microfluidic platform consisting of microchannels interconnected by a 3D ECM hydrogel, which enabled the real-time analysis of cancer cell invasion and intravasation into an endothelial monolayer.[37] Endothelial barrier function was found to be modulated by soluble biochemical factors and macrophages, which directly impacted the rate of tumor intravasation.[37] These attempts are however, limited in their ability to recreate the inherent characteristic properties of in vivo endothelia, due to the artificially-induced blood vessel morphogenesis. To overcome this problem, other microfluidic approaches have made important advances toward the formation of 3D capillary tubes in a more physiological manner, by inducing the in vitro formation of capillary networks as opposed to generating endothelial cell-lined channels, through the synergistic use of an alginate-based framework and medium perfusion.[39] A recent investigation by Kim et al. tried to recapitulate all these aspects within a perfusable microfluidic system, by combining heterotypic cell-cell interactions in 3D constructs together with the formation of interconnected networks of microvessels.[38] The high fidelity of this system resulted in vascular-specific response to shear stress of the endothelium, satisfactory recreation of angiogenesis and vasculogenesis, and perfusion of medium through the engineered microvessels.

In summary, this section demonstrates the broad applicability of microfluidic systems to model the manifold aspects of tumor progression. Notably, given the versatility offered by microfluidic systems, it is crucial to identify the fundamental parameters in each application so that the resulting microfluidic models are uniquely tailored to accurately reflect specific oncological mechanisms. For instance, while the co-culture of liver cells with cancer cells is necessary to simulate the dependency of anti-cancer drug efficacy on hepatic metabolism, the inclusion of liver cells may not be as critical in evaluating the migratory behavior of cancer cells in response to interstitial flow.[28,34]

\section{Macroscale Perfused Tumor Models}

Despite the exponential progress in the last decade, the so-called 'world-to-chip' problem continues to persist in the field of microfluidics.[40] Due to the inherent differences in scale, even the simplest microfluidic configuration may lead to differences in cellular response when compared to classical macroscale systems used for biological assays.[37,41] This discrepancy may be more apparent in modeling tumorigenesis, a highly complex process which engages both the micro- and macro-environment.[42] Accordingly, macroscale perfused tissue-engineered systems, such as flow perfusion bioreactors, may be a necessary affiliate to microfluidic culture systems in modeling tumor progression. However, given that the tumor engineering community is only beginning to appreciate the need for perfused macroscale models to mimic the biophysical properties of the tumor microenvironment and to overcome diffusional limitations,[1] literature in this area is still sparse. Amongst the few examples in the literature, Mishra et al. extrapolated the concept of organ reengineering to develop an ex vivo lung cancer model, by using a decellularized rat lung matrix as a scaffold to culture human lung cancer cells.[43] The authors demonstrated that the seeded lung cancer cells grown within the native matrix formed perfusable tumor nodules with similar 
features to the original human lung cancer, suggesting the applicability of this ex vivo model for mechanistic studies of lung cancer progression.

Other potentially translatable techniques developed for perfusing normal tissue constructs to tumor engineering are currently only at the conception stage. Perfused macroscale systems have seen success in modeling normal tissues such as musculoskeletal [44-46] and cardiovascular tissues [47], where they have been used to engineer uniform constructs at the millimeter-scale in the presence of physiological stimuli such as electrical stimulation, shear stress and native tissue contraction.[47] Notably, even though these systems do not offer the same degree of spatiotemporal control as their microfluidic counterparts, precise mathematical modeling can be employed to predict flow conditions and correlate the cellular response to system specifications. $[47,48]$ Leveraging similar porous polymeric scaffolds used for engineering perfusable normal tissues [46,49,50], the development of 3D biomimetic tumor models which recapitulate aspects of the tumor microenvironment has been reported.[51-53] In a landmark study, by culturing human oral squamous carcinoma cells within porous poly(lactic-co-glycolic acid) scaffolds, Fischbach et al. established a 3D engineered model that exhibits in vivo-like angiogenic characteristics and drug response.[51] More recently, our laboratory demonstrated the feasibility of employing scaffolds that are compatible with flow perfusion bioreactor systems and previously used for creating perfusable bone tissue constructs, to the development of in vitro bone tumors.[53] By culturing Ewing sarcoma cells within porous 3D electrospun poly(e-caprolactone) scaffolds, we observed that the tumor cells were not only more resistant to traditional cytotoxic drugs but also exhibited remarkable differences in the expression pattern of the insulin-like growth factor-1 receptor/mammalian target of rapamycin pathway as compared to cells cultured in 2D monolayer.[53]

Given that promising advances have been achieved by the tumor engineering community towards the development of in vivo-like tumor constructs, one can imagine that the incorporation of actual perfusion into these technically perfusable tissue-engineered tumor constructs would greatly enhance our understanding of the interplay between architectural, biochemical and biophysical cues and their impact on tumor progression. Notably, while bioreactors in tissue engineering often serve only to support an intermediate tissue construct that would eventually be implanted in vivo for further tissue maturation to occur, the ultimate goal in tumor engineering is to fully recreate the tumor tissue in vitro to derive a fundamental and accurate understanding of cancer biology. Hence, efforts to develop macroscale perfused cancer models should not only focus on translating current bioreactor strategies in tissue engineering to tumor engineering, but also in the adaptation of these strategies to account for the need to fully model the tumor microenvironment in vitro with high fidelity.

\section{Conclusions}

A microfluidic approach to the development of tissue-engineered, perfused tumor models is evidently dominant in the literature. The strong relevance of microfluidic cultures in oncology is not surprising, given the recognized molecular basis of cancer and the inherent capacity of these systems to precisely model biological processes occurring at the cellular level. As microfluidic approaches become progressively versatile in accommodating more advanced tissue-engineered scaffolds, one can expect that these miniature systems are likely to meet the mammoth challenge of synergistically integrating both biochemical and biophysical cues together, with the capability to model tumor vasculature and interstitial flow. 
On the other end, the hierarchical nature of tumorigenesis - evolving on multiple scales, from the subcellular to the organ level - has long been recognized, underscoring the importance of macroscale models as a necessary partner to those at the microscale. Furthermore, given the significant advances in tumor engineering in terms of recapitulating architectural cues and tumor-stroma interactions at the macroscale, a logical progression in this field is the introduction of perfusion strategies to more fully mimic the tumor microenvironment.

With both micro- and macro-approaches currently advancing in parallel and exchanging technology, one can envisage an ultimate convergence toward the development of perfused, in vivo-like tissues in vitro, potentially resulting in paradigm-changing breakthroughs in the evaluation of drug efficacy and mechanistic studies of tumor biology.

\section{Acknowledgments}

Funding support has been provided by the National Institutes of Health, under the grants R01 AR057083 and P01 CA98912. Fong E.L. acknowledges funding from the National University of Singapore - Overseas Graduate Scholarship.

\section{References}

1. Burdett E, Kasper FK, Mikos AG, Ludwig JA. Engineering tumors: A tissue engineering perspective in cancer biology. Tissue Eng Part B-Re. 2010; 16(3):351-359.

2. Gurski LA, Xu X, Labrada LN, Nguyen NT, Xiao L, van Golen KL, Jia X, Farach-Carson MC. Hyaluronan (ha) interacting proteins rhamm and hyaluronidase impact prostate cancer cell behavior and invadopodia formation in 3d ha-based hydrogels. PLoS One. 2012; 7(11):e50075. [PubMed: 23166824]

3. Koumoutsakos P, Pivkin I, Milde F. The fluid mechanics of cancer and its therapy. Annu Rev Fluid Mech. 2013; 45:325-355.

4. Hagendoorn J, Tong R, Fukamura D, Lin QC, Lobo L, Padera TP, Xu L, Kucherlapati R, Jain RK. Onset of abnormal blood and lymphatic vessel function and interstitial hypertension in early stages of carcinogenesis. Cancer Res. 2006; 66(7):3360-3364. [PubMed: 16585153]

5. Rutkowski JM, Swartz MA. A driving force for change: Interstitial flow as a morphoregulator. Trends in cell biology. 2007; 17(1):44-50. [PubMed: 17141502]

6 . Boucher Y, Jain RK. Microvascular pressure is the principal driving force for interstitial hypertension in solid tumors: Implications for vascular collapse. Cancer Res. 1992; 52(18):51105114. [PubMed: 1516068]

7. Chang SF, Chang CA, Lee DY, Lee PL, Yeh YM, Yeh CR, Cheng CK, Chien S, Chiu JJ. Tumor cell cycle arrest induced by shear stress: Roles of integrins and smad. Proc Natl Acad Sci U S A. 2008; 105(10):3927-3932. [PubMed: 18310319]

8. Fleury ME, Boardman KC, Swartz MA. Autologous morphogen gradients by subtle interstitial flow and matrix interactions. Biophysical journal. 2006; 91(1):113-121. [PubMed: 16603487]

9. Shields JD, Fleury ME, Yong C, Tomei AA, Randolph GJ, Swartz MA. Autologous chemotaxis as a mechanism of tumor cell homing to lymphatics via interstitial flow and autocrine ccr7 signaling. Cancer cell. 2007; 11(6):526-538. [PubMed: 17560334]

10•. Munson JM, Bellamkonda RV, Swartz MA. Interstitial flow in a $3 \mathrm{~d}$ microenvironment increases glioma invasion by a cxcr4-dependent mechanism. Cancer Res. 2013; 73(5):1536-1546. The effects of interstitial flow on glioma cell invasion were investigated in this study, where it was reported that the effects observed were dependent on CXCR4. This study describes the mechanisms for tumor invasion - interstitial flow-enhanced motility and chemokine-driven autologous chemotaxis. [PubMed: 23271726]

11. Velve-Casquillas G, Le Berre M, Piel M, Tran PT. Microfluidic tools for cell biological research. Nano Today. 2010; 5(1):28-47. [PubMed: 21152269]

Curr Opin Chem Eng. Author manuscript; available in PMC 2015 February 01. 
12. Li X, Chen Y, Li PCH. A simple and fast microfluidic approach of same-single-cell analysis (sasca) for the study of multidrug resistance modulation in cancer cells. Lab on a Chip. 2011; 11(7):1378-1384. [PubMed: 21327253]

13. Wlodkowic D, Cooper JM. Tumors on chips: Oncology meets microfluidics. Curr Opin Chem Biol. 2010; 14(5):556-567. [PubMed: 20832352]

14. Chikkaveeraiah BV, Mani V, Patel V, Gutkind JS, Rusling JF. Microfluidic electrochemical immunoarray for ultrasensitive detection of two cancer biomarker proteins in serum. Biosens Bioelectron. 2011; 26(11):4477-4483. [PubMed: 21632234]

15. Autebert J, Coudert B, Bidard FC, Pierga JY, Descroix S, Malaquin L, Viovy JL. Microfluidic: An innovative tool for efficient cell sorting. Methods. 2012; 57(3):297-307. [PubMed: 22796377]

16. Sung JH, Shuler ML. A micro cell culture analog (microcca) with 3-d hydrogel culture of multiple cell lines to assess metabolism-dependent cytotoxicity of anti-cancer drugs. Lab Chip. 2009; 9(10): 1385-1394. [PubMed: 19417905]

17. Jang Y-H, Hancock MJ, Kim SB, Selimovic S, Sim WY, Bae H, Khademhosseini A. An integrated microfluidic device for two-dimensional combinatorial dilution. Lab on a Chip. 2011; 11(19): 3277-3286. [PubMed: 21837312]

18. Ghaemmaghami AM, Hancock MJ, Harrington H, Kaji H, Khademhosseini A. Biomimetic tissues on a chip for drug discovery. Drug Discovery Today. 2012; 17(3-4):173-181. [PubMed: 22094245]

19. Delnero P, Song YH, Fischbach C. Microengineered tumor models: Insights \& opportunities from a physical sciences-oncology perspective. Biomed Microdevices. 2013; 15(4):583-593. [PubMed: 23559404]

20•. Du Y, Ghodousi M, Qi H, Haas N, Xiao W, Khademhosseini A. Sequential assembly of cell-laden hydrogel constructs to engineer vascular-like microchannels. Biotechnol Bioeng. 2011; 108(7): 1693-1703. This paper presents an innovative method for the fabrication of a fully perfusable interconnected construct, with negligible effects on cell viability, low cost and high reproducibility. [PubMed: 21337336]

21. Shin MK, Kim SK, Jung H. Integration of intra- and extravasation in one cell-based microfluidic chip for the study of cancer metastasis. Lab Chip. 2011; 11(22):3880-3887. [PubMed: 21975823]

22. Dickinson LE, Lutgebaucks C, Lewis DM, Gerecht S. Patterning microscale extracellular matrices to study endothelial and cancer cell interactions in vitro. Lab Chip. 2012; 12(21):4244-4248. [PubMed: 22992844]

23. Buchanan C, Rylander MN. Microfluidic culture models to study the hydrodynamics of tumor progression and therapeutic response. Biotechnol Bioeng. 2013; 110(8):2063-2072. [PubMed: 23616255]

24. Chen MC, Gupta M, Cheung KC. Alginate-based microfluidic system for tumor spheroid formation and anticancer agent screening. Biomed Microdevices. 2010; 12(4):647-654. [PubMed: 20237849]

25. Jeon JS, Zervantonakis IK, Chung S, Kamm RD, Charest JL. In vitro model of tumor cell extravasation. PLoS One. 2013; 8(2):e56910. [PubMed: 23437268]

26. Sung KE, Yang N, Pehlke C, Keely PJ, Eliceiri KW, Friedl A, Beebe DJ. Transition to invasion in breast cancer: A microfluidic in vitro model enables examination of spatial and temporal effects. Integr Biol (Camb). 2011; 3(4):439-450. [PubMed: 21135965]

27. Huang CP, Lu J, Seon H, Lee AP, Flanagan LA, Kim HY, Putnam AJ, Jeon NL. Engineering microscale cellular niches for three-dimensional multicellular co-cultures. Lab Chip. 2009; 9(12): 1740-1748. [PubMed: 19495458]

28. Polacheck WJ, Charest JL, Kamm RD. Interstitial flow influences direction of tumor cell migration through competing mechanisms. Proc Natl Acad Sci U S A. 2011; 108(27):11115-11120. [PubMed: 21690404]

29••. Haessler U, Teo JC, Foretay D, Renaud P, Swartz MA. Migration dynamics of breast cancer cells in a tunable $3 \mathrm{~d}$ interstitial flow chamber. Integr Biol (Camb). 2012; 4(4):401-409. A comprehensive investigation of the effect of interstitial flow on tumor cell migration. By mimicking interstitial flow in a microfluidic device, this study illustrates the heterogeneity of 
tumor cell migration in response to interstitial flow, a phenomenon previously not reported in studies with end-point or averaged measurements. [PubMed: 22143066]

30. Altmann B, Welle A, Giselbrecht S, Truckenmuller R, Gottwald E. The famous versus the inconvenient - or the dawn and the rise of 3d-culture systems. World journal of stem cells. 2009; 1(1):43-48. [PubMed: 21607106]

31. Slaughter BV, Khurshid SS, Fisher OZ, Khademhosseini A, Peppas NA. Hydrogels in regenerative medicine. Advanced materials. 2009; 21(32-33):3307-3329. [PubMed: 20882499]

32. Toh YC, Zhang C, Zhang J, Khong YM, Chang S, Samper VD, van Noort D, Hutmacher DW, Yu H. A novel 3d mammalian cell perfusion-culture system in microfluidic channels. Lab Chip. 2007; 7(3):302-309. [PubMed: 17330160]

33. Ong SM, Zhang C, Toh YC, Kim SH, Foo HL, Tan CH, van Noort D, Park S, Yu H. A gel-free 3d microfluidic cell culture system. Biomaterials. 2008; 29(22):3237-3244. [PubMed: 18455231]

34. Ma L, Barker J, Zhou C, Li W, Zhang J, Lin B, Foltz G, Kublbeck J, Honkakoski P. Towards personalized medicine with a three-dimensional micro-scale perfusion-based two-chamber tissue model system. Biomaterials. 2012; 33(17):4353-4361. [PubMed: 22429982]

35. Stroock AD, Fischbach C. Microfluidic culture models of tumor angiogenesis. Tissue engineering Part A. 2010; 16(7):2143-2146. [PubMed: 20214470]

36••. Zheng Y, Chen J, Craven M, Choi NW, Totorica S, Diaz-Santana A, Kermani P, Hempstead B, Fischbach-Teschl C, Lopez JA, Stroock AD. In vitro microvessels for the study of angiogenesis and thrombosis. Proc Natl Acad Sci U S A. 2012; 109(24):9342-9347. This paper describes the formation of microvascular channels in a 3D scaffold with enhanced recapitulation of microvasculature physiology. The engineered vascular network mimics aspects of healthy as well as pathological endothelia, angiogenesis and the inflammatory response. [PubMed: 22645376]

37•. Zervantonakis IK, Hughes-Alford SK, Charest JL, Condeelis JS, Gertler FB, Kamm RD. Threedimensional microfluidic model for tumor cell intravasation and endothelial barrier function. Proc Natl Acad Sci U S A. 2012; 109(34):13515-13520. Using a microfluidic device to mimic the tumor-vasculature interface, the authors demonstrate that the endothelium serves as a barrier to tumor cell intravasation, which can be modulated by factors (such as interactions with macrophages) present in the tumor microenvironment. [PubMed: 22869695]

38. Kim S, Lee H, Chung M, Jeon NL. Engineering of functional, perfusable 3d microvascular networks on a chip. Lab Chip. 2013; 13(8):1489-1500. [PubMed: 23440068]

39. Chan JM, Zervantonakis IK, Rimchala T, Polacheck WJ, Whisler J, Kamm RD. Engineering of in vitro 3d capillary beds by self-directed angiogenic sprouting. PLoS One. 2012; 7(12):e50582. [PubMed: 23226527]

40. Kobel S, Lutolf M. High-throughput methods to define complex stem cell niches. Biotechniques. 2010; 48(4):ix-xxii. [PubMed: 20569203]

41. Paguirigan AL, Beebe DJ. From the cellular perspective: Exploring differences in the cellular baseline in macroscale and microfluidic cultures. Integr Biol (Camb). 2009; 1(2):182-195. [PubMed: 20023802]

42. Bissell MJ, Radisky D. Putting tumours in context. Nat Rev Cancer. 2001; 1(1):46-54. [PubMed: 11900251]

43. Mishra DK, Thrall MJ, Baird BN, Ott HC, Blackmon SH, Kurie JM, Kim MP. Human lung cancer cells grown on acellular rat lung matrix create perfusable tumor nodules. The Annals of thoracic surgery. 2012; 93(4):1075-1081. [PubMed: 22385822]

44. Liao J, Guo X, Grande-Allen KJ, Kasper FK, Mikos AG. Bioactive polymer/extracellular matrix scaffolds fabricated with a flow perfusion bioreactor for cartilage tissue engineering. Biomaterials. 2010; 31(34):8911-8920. [PubMed: 20797784]

45. Grayson WL, Marolt D, Bhumiratana S, Frohlich M, Guo XE, Vunjak-Novakovic G. Optimizing the medium perfusion rate in bone tissue engineering bioreactors. Biotechnol Bioeng. 2011; 108(5):1159-1170. [PubMed: 21449028]

46. Thibault RA, Mikos AG, Kasper FK. Scaffold/extracellular matrix hybrid constructs for bonetissue engineering. Adv Healthc Mater. 2013; 2(1):13-24. [PubMed: 23184883] 
47. Maidhof R, Tandon N, Lee EJ, Luo J, Duan Y, Yeager K, Konofagou E, Vunjak-Novakovic G. Biomimetic perfusion and electrical stimulation applied in concert improved the assembly of engineered cardiac tissue. J Tissue Eng Regen Med. 2012; 6(10):e12-23. [PubMed: 22170772]

48. Kapellos GE, Alexiou TS, Payatakes AC. Theoretical modeling of fluid flow in cellular biological media: An overview. Math Biosci. 2010; 225(2):83-93. [PubMed: 20303992]

49. Woodruff MA, Lange C, Chen F, Fratzl P, Hutmacher DW. Nano- to macroscale remodeling of functional tissue-engineered bone. Adv Healthc Mater. 2013; 2(4):546-551. [PubMed: 23184872]

50. Sikavitsas VI, Bancroft GN, Holtorf HL, Jansen JA, Mikos AG. Mineralized matrix deposition by marrow stromal osteoblasts in $3 \mathrm{~d}$ perfusion culture increases with increasing fluid shear forces. Proc Natl Acad Sci U S A. 2003; 100(25):14683-14688. [PubMed: 14657343]

51. Fischbach C, Chen R, Matsumoto T, Schmelzle T, Brugge JS, Polverini PJ, Mooney DJ. Engineering tumors with 3d scaffolds. Nature methods. 2007; 4(10):855-860. [PubMed: 17767164]

52. Fischbach C, Kong HJ, Hsiong SX, Evangelista MB, Yuen W, Mooney DJ. Cancer cell angiogenic capability is regulated by $3 \mathrm{~d}$ culture and integrin engagement. Proc Natl Acad Sci U S A. 2009; 106(2):399-404. [PubMed: 19126683]

53•. Fong EL, Lamhamedi-Cherradi SE, Burdett E, Ramamoorthy V, Lazar AJ, Kasper FK, FarachCarson MC, Vishwamitra D, Demicco EG, Menegaz BA, Amin HM, et al. Modeling ewing sarcoma tumors in vitro with 3d scaffolds. Proc Natl Acad Sci U S A. 2013; 110(16):6500-6505. By using a porous polymeric scaffold, the authors report the establishment of an in vitro model of bone sarcoma, with in vivo-like morphology, protein expression and drug response. Similar scaffolds have been employed within bioreactors and the presence of fluid flow to create bone tissue constructs. [PubMed: 23576741] 


\section{Highlights}

- Role of fluid flow in cancer progression remains inadequately elucidated.

- Recapitulation of fluid dynamics requires both microfluidic and macroscale models.

- Microfluidic models enable oncology investigations at the single-cell level.

- Macroscale models can reproducibly mimic the hierarchical nature of tumorigenesis. 

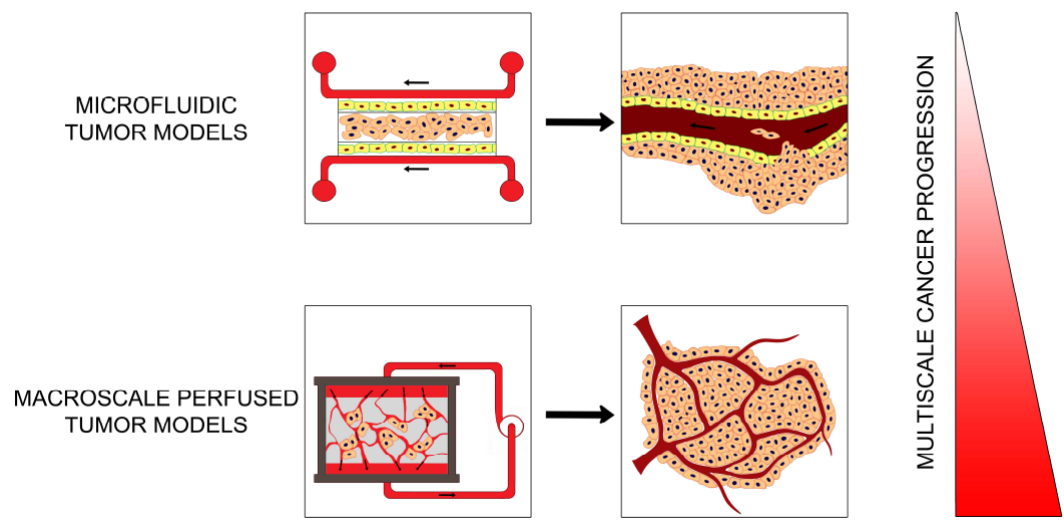

Figure 1.

Hierarchical nature of cancer progression. The integrated use of microfluidic and macroscale perfused models may synergistically enhance our understanding of fluid dynamics in tumorigenesis on multiscales. 